\title{
Avaliação funcional pós-operatória em pacientes submetidos à técnica de Sauvé-Kapandji
}

Postoperative functional evaluation in patients undergoing Sauvé-Kapandji procedure Evaluación funcional postoperatoria en pacientes sometidos al procedimiento de Sauvé-Kapandji \author{
Caroline Brum SENA ${ }^{\mathbf{1}}$ \\ Filipe Jun SHIMAOKA ${ }^{2}$ \\ Luis Guilherme Rosifini Alves REZENDE ${ }^{2}$ \\ Luiz Garcia MANDARANO-FILHO ${ }^{2}$
}

Nilton MAZZER ${ }^{3}$

${ }^{1}$ Cirurgiã da Mão, formada pelo Programa de Cirurgia da Mão do Hospital das Clínicas da Faculdade de Medicina de Ribeirão Preto da Universidade de São Paulo, HC-FMRP-USP, Campus Monte Alegre, 14049-900 Ribeirão Preto - SP, Brasil

${ }^{2}$ Médico(a) Assistente do Programa de Cirurgia da Mão, Hospital das Clínicas da Faculdade de Medicina de Ribeirão Preto da Universidade de São Paulo, HC-FMRP-USP, Campus Monte Alegre, 14049-900 Ribeirão Preto - SP, Brasil

${ }^{3}$ Professor Titular e Chefe da Divisão de Cirurgia da Mão, Hospital das Clínicas da Faculdade de Medicina de Ribeirão Preto da Universidade de São Paulo, HC-FMRP-USP, Campus Monte Alegre, 14049-900 Ribeirão Preto - SP, Brasil

\section{Resumo}

Introdução: O acometimento da articulação radioulnar distal ocasiona um importante comprometimento funcional da mão, sendo um ponto crítico para a manutenção da estabilidade do antebraço, causando um grande impacto na qualidade de vida desta população. Objetivo: avaliar os resultados funcionais dos pacientes operados pela técnica de Sauvé-Kapandji. Materiais e Métodos: Estudo retrospectivo, pela avaliação de 16 pacientes já operados pela técnica de pós-operatório da técnica de Sauvé- Kapandji em acompanhamento no ambulatório. Foram avaliadas radiografias pré e pós-operatórias, teste da função de pinça, questionário PRWE. Resultados: Foram regulares e satisfatórios (62,25\%). A taxa de reoperação foi de $37,5 \%$ para retirada dos implantes. Não houve diferença entre as radiografias sem e com carga $(p=0,365)$. A dor após a cirurgia foi de 3,1 \pm 0,8. Conclusão: Os resultados foram satisfatórios indicando bom prognóstico para os pacientes com alteração na articulação radioulnar operados pela técnica de Sauvé-Kapandji.

Descritores: Articulação do Punho; Artrodese; Dor.

\section{Abstract}

Introduction: The involvement of the distal radioulnar joint causes an important functional impairment of the hand, being a critical point for maintaining the stability of the forearm, causing a great impact on the quality of life of this population. Objective: to evaluate the functional results of patients operated on using the Sauvé-Kapandji procedure. Materials and Methods: Retrospective study, by evaluating 16 patients already operated on by the post-operative technique of the Sauvé-Kapandji procedure being followed up at the outpatient clinic. Pre and postoperative radiographs, clamp function test, PRWE questionnaire were evaluated. Results: They were regular and satisfactory $(62.25 \%)$. The reoperation rate was $37.5 \%$ for removal of the implants. There was no difference between radiographs without and with load $(p=0.365)$. Pain after surgery was $3.1 \pm 0.8$. Conclusion: The results were satisfactory, indicating a good prognosis for patients with alterations in the radioulnar joint operated by the Sauvé-Kapandji procedure.

Descriptors: Wrist Joint; Arthrodesis; Pain.

\section{Resumen}

Introducción: La afectación de la articulación radiocubital distal provoca un importante deterioro funcional de la mano, siendo un punto crítico para mantener la estabilidad del antebrazo, provocando un gran impacto en la calidad de vida de esta población. Objetivo: evaluar los resultados funcionales de los pacientes operados con lo procedimiento de Sauvé-Kapandji. Materiales y Métodos: Estudio retrospectivo, evaluando 16 pacientes que ya habían sido sometidos a la técnica postoperatoria de la técnica Sauvé-Kapandji en seguimiento en la consulta externa. Se evaluaron radiografías pre y postoperatorias, prueba de función de clamp, cuestionario PRWE. Resultados: Fueron regulares y satisfactorios (62,25\%). La tasa de reintervención fue del $37,5 \%$ para la extracción de los implantes. No hubo diferencia entre las radiografías cargadas y descargadas $(p=0,365)$. El dolor postoperatorio fue de 3,1 \pm 0,8 Conclusión: Los resultados fueron satisfactorios, lo que indica un buen pronóstico para los pacientes con alteraciones en la articulación radiocubital intervenidos mediante lo procedimiento de Sauvé-Kapandji.

Descriptores: Articulación de la Muñeca; Artrodesis; Dolor.

INTRODUÇÃO

As doenças degenerativas da articulação radioulnar distal, seja qual for sua etiologia, ocasionam um importante comprometimento funcional da mão. A integridade desta articulação é o ponto crítico para a manutenção da estabilidade do punho e antebraço, afetando significativamente a capacidade de pronação e supinação. A sintomatologia também inclui dor, fraqueza e dificuldades na execução das atividades da vida diária, gerando grande impacto na qualidade de vida dos pacientes. Por esta razão, um tratamento adequado é necessário para restabelecer essas perdas de forma parcial ou total ${ }^{1-5}$.

A degeneração da articulação radioulnar podem ser divididas em três grandes grupos: lesões traumáticas, como sequelas das fraturas da extremidade distal do rádio ou do antebraço; degenerativas, como a artrite reumatoide; e congênitas, por exemplo, a doença de Madelung. Vários procedimentos cirúrgicos foram propostos para o tratamento desta afecção, mais notoriamente a técnica de Bowers, Darrach e Sauvé-Kapandji. As limitações destes métodos são a instabilidade da articulação radioulnar distal, o impacto da ulnocarpal e, consequentemente, a dor residual no punho. Apesar de várias opções, ainda não se sabe qual é a mais adequada ou eficaz, sendo ainda um ponto de interesse na literatura ${ }^{3-12}$.

Sauvé e Kapandji, em 1936 2,3,10, descreveram uma técnica que consiste na fusão da articulação radioulnar distal por cruentizarção 
das superfícies articulares e fixação com fios de Kirschner ou parafuso; associando a ressecção de um segmento de 10 a $20 \mathrm{~mm}$ da metáfise distal da ulna para que, neste local, se desenvolva uma pseudo-artrose ${ }^{2,11}$. O método, conhecido como procedimento de SauvéKapandji, é indicado para tratar os desarranjos da articulação radioulnar distal que resultem em incongruência, instabilidade e dor, refratárias ao tratamento não operatório ${ }^{13-16}$. Também é considerada uma cirurgia de salvação após procedimentos cirúrgicos mal sucedidos como a hemirressecção-interposição de Bowers ${ }^{9}$. As principais contraindicações são a artrite séptica ativa, a ausência da cabeça do radio e o comprimento insuficiente da ulna, este ultimo comum após o procedimento de Darrach ou a ressecção tumoral ${ }^{2}$.

A operação de Sauvé-Kapandji ganhou muitos adeptos nos últimos anos, face aos bons resultados obtidos, principalmente pelo alívio da dor e pelo ganho da prono-supinação ${ }^{8,11}$. Inúmeras vantagens têm sido relatadas, sobretudo quando comparadas à cirurgia de Darrach. Assim, a presença da cabeça da ulna propicia a permanência dos contornos estéticos do punho, impede a luxação do tendão flexor ulnar do carpo, e conserva a transmissão fisiológica de forças entre a mão e o antebraço, pelo contato ulnocarpal ${ }^{2,15-19}$. Por outro lado, a principal desvantagem, ou complicação, é a instabilidade do segmento proximal da ulna ${ }^{20-24}$.

O objetivo deste estudo foi avaliar funcionalmente, restropectivamente, pacientes operados pela técnica de Sauvé-Kapandji, em um hospital terciário no interior do estado de São Paulo, Brasil.

MATERIAL E MÉTODO

Trata-se de estudo descritivo, retrospectivo, transversal com abordagem quantitativa, realizado no Hospital das Clínicas da Faculdade de Medicina de Ribeirão Preto da Universidade de São Paulo - SP, Brasil. A região metropolitana de Ribeirão Preto, criada em 2016, abrange 34 municípios, com população de 1.738 .000 habitantes segundo dados da Pesquisa Demográfica Médica no Estado de São Paulo de 2020. O projeto foi aprovado pela Comissão de Ética em Pesquisa da Faculdade de Medicina de Ribeirão Preto da Universidade de São Paulo (FMRP-USP) sob número 47697015.2.0000.5440.

Foram avaliados funcionalmente pacientes submetidos ao procedimento de Sauvé-Kapandji entre 2010 a 2015, que se encontravam aos 12 meses do período pósoperatório superior. Os critérios de exclusão foram pacientes com período pós-operatório inferior a 12 meses ou que não compareceram nos retornos estabelecidos. A amostra consistiu em 16 pacientes, sendo 8 homens e 8 mulheres.

A técnica cirúrgica consistiu em anestesia do tipo sedação associada ao bloqueio regional. Realizou-se uma incisão longitudinal dorsal sobre a ulna, expondo a ARUD, que foi cruentizada até a visualização de osso esponjoso. Realizou-se a ressecção de $2,0 \mathrm{~cm}$ de osso na transição metadiafisária distal da ulna, com serras sagitais. Em seguida, a cabeça da ulna foi posicionada e fixada ao rádio com parafuso (3.5mm) de compressão interfragmentária (Figura 1). Associou-se a estabilização do segmento proximal da ulna com uma hemi-fita do tendão extensor ulnar do carpo.

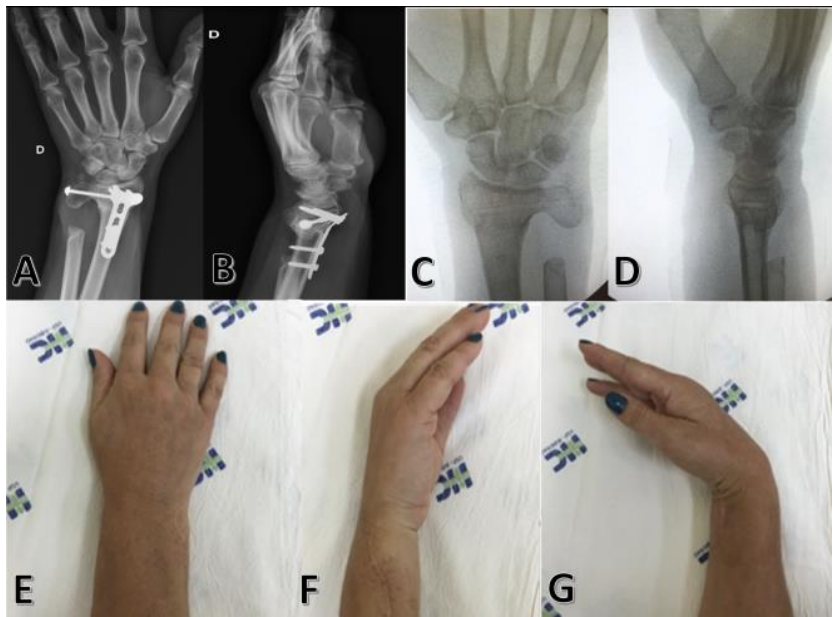

Figura 1: A-B: Paciente de 44 anos com sequela de fratura de radio distal. Radiografia da osteotomia corretiva do radio e SauvéKapandji. C-D: Radiografia após três anos de retirada do material. E-G: Função da paciente (Fonte: HC-FMRP-USP).

A análise de imagem foi realizada aos 12 meses pós-operatórios com radiografia em anteroposterior e perfil, sem carga e com carga de $3 \mathrm{~kg}$ no membro superior (lado operado), segurados pelo paciente durante o exame (Figura 2).

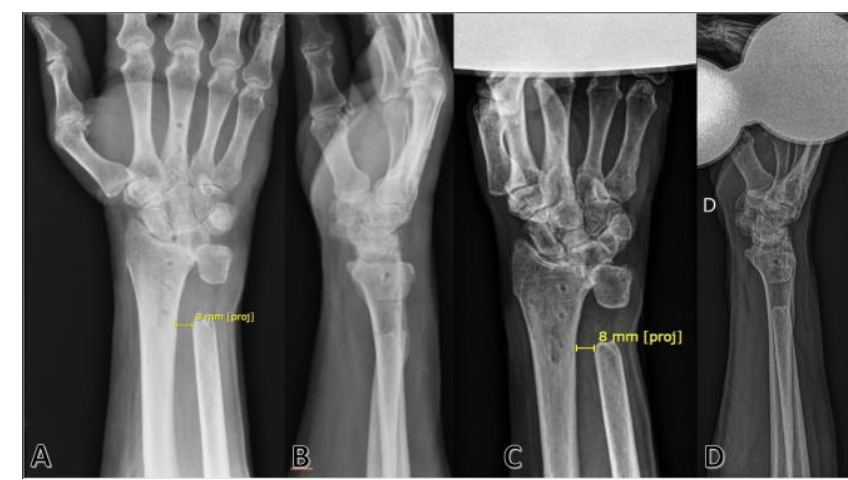

Figura 2: Radiografias em anteroposterior e perfil no período pós operatório. A e B: sem carga. C e D: com carga de $3 \mathrm{~kg}$ (Fonte: HC-FMRP-USP).

A avaliação física foi composta pela análise de força de preensão da mão com método de Grip e Pinch, utilizando um 
dinamômetro de Jamar e registradi em $\mathrm{Kg}$ (Figura 3). Os dados funcionais foram avaliados através do questionário PRWE (Patient Rated Wrist Evaluation) e escala análoga da dor.

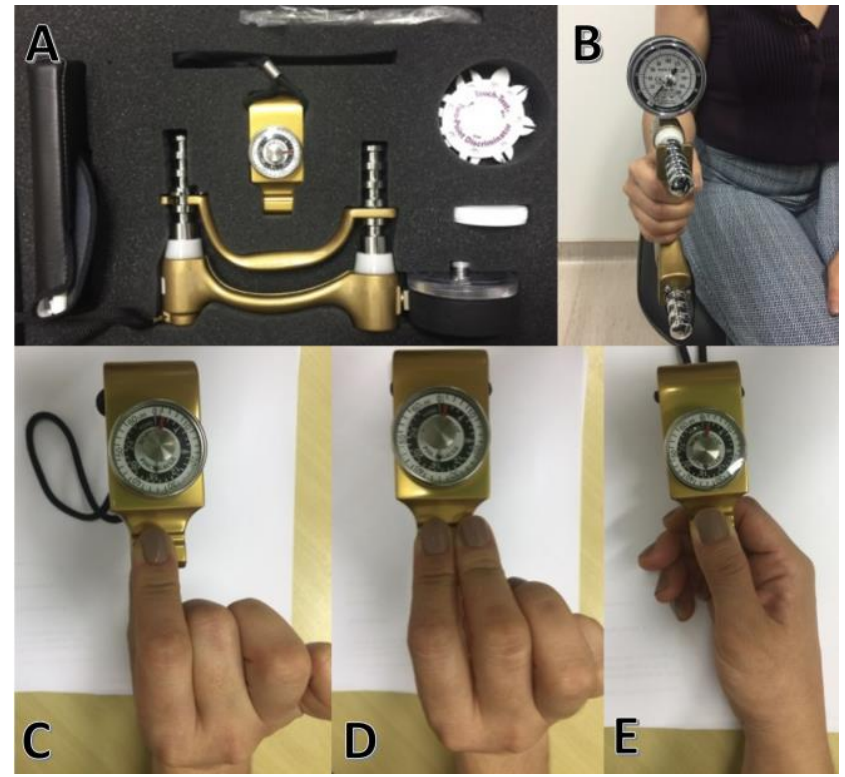

Figura 3: A: Equipamento completo. B: Dinamômetro de Jamar. C: Teste ponta a ponta. D: Teste trípode. E: Teste de Pinch (Fonte: HC-FMRP-USP).

Para avaliar os parâmetros intrínsecos da amostragem quanto à normalidade e distribuição foi utilizado o teste de KolmogorovSmirnov. Os dados foram expressos em média, desvio padrão e porcentagem (SPSS Statistical software). As variáveis foram analisadas descritivamente através da média, desviopadrão, mínimos e máximos e Intervalos de Confiança a $95 \%$. O estudo da diferença entre as médias de duas variáveis foi estabelecido pelo teste-t de student e o índice de correlação através do coeficiente de correlação de Pearson. O nível de significância utilizado para os testes foi de $5 \%$.

RESULTADOS

A idade média foi de 48 anos, obtendose resultado satisfatório em $62,25 \%$ dos pacientes. Todos os pacientes possuíam sequelas de fraturas do rádio distal, consistindo em 8 possuíam lesões no lado direito e 8 no lado esquerdo. O tempo cirúrgico médio foi de 60 minutos. Os pacientes em sua maioria eram estudantes e aposentados, e os demais exerciam funções laborais com esforço do punho. Para descaracterizar possíveis viéses de interferências em relação ao sexo e idade, o teste de correlação de Pearson foi determinado. Não houve associação positiva entre as variáveis, como é possível observar na Tabela 1.

Não houve complicações como infecção, não consolidação da artrodese ou crescimento ósseo da ulna no segmento ressecado. A única complicação relatada foi a irritação do implante, havendo necessidade de retirada do parafuso em $37,75 \%(n=6)$ dos casos. A reabilitação foi iniciada no pós-operatório imediato para analgesia e contenção de edemas locais. Após cicatrização da pele, o paciente foi submetido à cinesioterapia para melhorar posicionamento do membro afetado, diminuir rigidez articular, ganhar força e mobilidade, além de estimular a parte sensorial e funcional. A evolução no tratamento foi dependente da melhora clínica individual. Por meio dos dados radiográficos foi possível observar uma diferença significativa entre o membro operado e contralateral em todas as avaliações, conforme a Tabela 2 e Figura 4. Porém, quando comparadas as radiografias sem carga $(m=8,99 \mathrm{dp}=2,75)$ e com carga $(m=9,52 d p=3,52)$, não se observou diferença significativa $(p=0,365)$.

Tabela 1. Teste de correlação de Pearson para descaracterizar correlação entre idade, sexo e tipos de avaliações.

\begin{tabular}{l|l|l|l|l}
\hline \multicolumn{2}{c|}{ Medidas } & Radigráfica & $\begin{array}{l}\text { Avaliação } \\
\text { Fisica }\end{array}$ & $\begin{array}{l}\text { Avaliação } \\
\text { Funcional }\end{array}$ \\
\hline \multirow{2}{*}{ Idade } & Pearson Correlation & 0,124 & 0,049 & 0,159 \\
\cline { 2 - 5 } & Valor p & 0,392 & 0,735 & 0,116 \\
\hline Sexo & Person Correlation & $-0,094$ & $-0,064$ & $-0,225$ \\
\hline & Valor p & 0,387 & 0,516 & 0,657 \\
\hline TOTAL & N & 16 & 16 & 16 \\
\hline
\end{tabular}

Tabela 2. Representação das medidas angulares em pacientes operados pela técnica de Sauvé- Kapandji. Fonte: Dados da Pesquisa

\begin{tabular}{|c|c|c|c|c|c|c|c|c|c|c|}
\hline \multicolumn{5}{|c|}{ OP } & \multicolumn{6}{|c|}{ DP } \\
\hline & MX & MN & M & DP & MX & MN & M & DP & $\begin{array}{l}\text { IC } \\
(95 \%)\end{array}$ & $p$ \\
\hline DR & 20 & o & 10 & 6,94 & 20 & 10 & 20 & 2,98 & $\begin{array}{l}5,72 \text { a } \\
13,48\end{array}$ & 0,0001 \\
\hline DU & 30 & 0 & 17,33 & 12,23 & 30 & 10 & 27 & 6,49 & $\begin{array}{l}9,51 \mathrm{a} \\
41,15\end{array}$ & 0,0065 \\
\hline FL & 80 & 0 & 47,67 & 32,78 & 80 & 45 & 73 & 12,51 & $\begin{array}{c}0,5590 \text { a } \\
8,78\end{array}$ & 0,004 \\
\hline EXT & 70 & 0 & 38,67 & 29,55 & 70 & 40 & 61,6 & 15,38 & $\begin{array}{l}3,17 a \\
16,16\end{array}$ & 0,047 \\
\hline PR & 90 & 70 & 84,6 & 8,33 & 90 & 90 & 89,3 & 2,58 & $\begin{array}{c}8,06 \text { a } \\
36,6\end{array}$ & 0,029 \\
\hline SUP & 90 & 0 & 72 & 31,44 & 90 & 90 & 90 & 0 & 0,53 & 0,043 \\
\hline
\end{tabular}

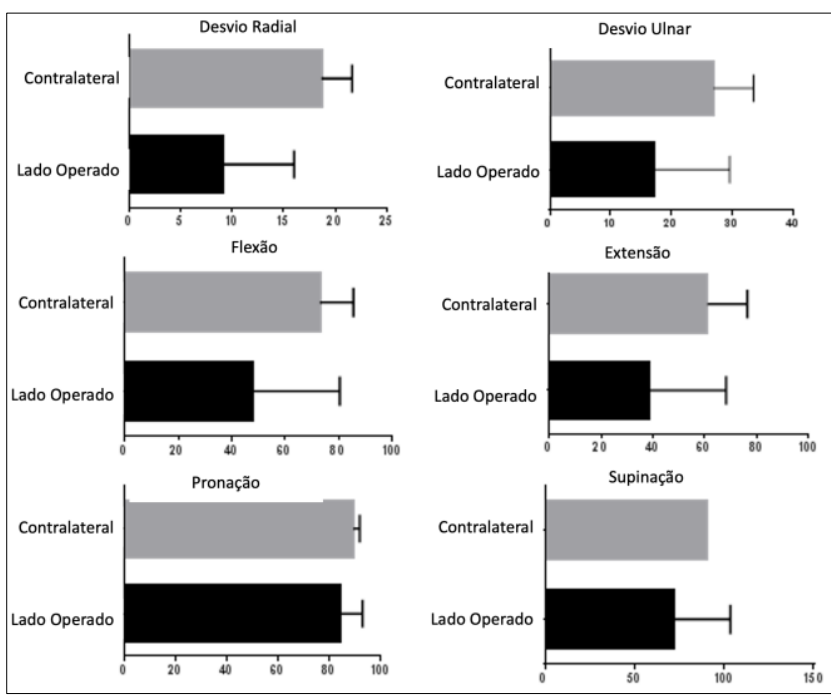

Figura 4: Representação gráfica dos ângulos medidos entre lado operado e contralateral Kapandji. Fonte: Dados da Pesquisa

$\mathrm{Na}$ avaliação física observa-se que houve uma diminuição da força do membro operado quando comparado ao membro contralateral (Tabela 3 e Figura 5). Por meio do 
questionário PRWE foi possível avaliar os critérios subjetivos da técnica de SauvéKapandji, notando-se uma melhora significativa nos pacientes para a avaliação de dor. Entretanto, a função apresentou um resultado inverso com piora pós-operatória. Estes parâmetros não alteraram a atividade pré e pósoperatória (Tabela 4 e Figura 6). Para saber se a satisfação do paciente era condizente com os resultados clínicos e funcionais coletados, os mesmos foram questionados para determinar uma nota de "0 a 10", ("0" equivalente a ausência de satisfação ou ausência de dor) para a cirurgia e para a dor. A nota média para a cirurgia foi $8,6 \pm 2,52$ e para dor $3,46 \pm 2,85$. A dor média relatada antes da cirurgia foi $9,2 \pm$ 1,31. Além disso, a correlação entre os dados funcionais e a avaliação dos pacientes foi positiva, segundo índice de correlação de Pearson $(r=0,892, p=0,002)$.

Tabela 3. Representação das medidas físicas e de força avaliadas nos pacientes operados. Fonte: Dados da Pesquisa

\begin{tabular}{|c|c|c|c|c|}
\hline \multicolumn{5}{|l|}{ DIREITA } \\
\hline & Máximo & Mínimo & Média & Desvio Padrão \\
\hline Jamar & 38 & 2,67 & 15,47 & 11,85 \\
\hline Tripode & 6,97 & 2 & 4,6 & 1,58 \\
\hline Pinch & 10,31 & 3,34 & 6,44 & 2,48 \\
\hline Pinça ponta a ponta & 5,87 & 1,34 & 3,77 & 1,29 \\
\hline ESQUERDA & & & & \\
\hline & Máximo & Mínimo & Média & Desvio Padrão \\
\hline Jamar & 33 & 2,24 & 15,30 & 10,61 \\
\hline Tripode & 7 & 1,95 & 4,59 & 1,78 \\
\hline Pinch & 10,41 & 3,21 & 6,57 & 2,17 \\
\hline Pinça ponta a ponta & 6,67 & 1,32 & 3,63 & 1,51 \\
\hline IC (95\%) & & & & \\
\hline Jamar & & & a 7,78 & \\
\hline Tripode & & -2, & a 2,36 & \\
\hline Pinch & & & 1,02 & \\
\hline Pinça ponta a ponta & & -1, & a 1,44 & \\
\hline $\boldsymbol{p}$ & & & & \\
\hline Jamar & & & 99 & \\
\hline Tripode & & & 85 & \\
\hline Pinch & & & 89 & \\
\hline Pinça ponta a ponta & & & 79 & \\
\hline
\end{tabular}

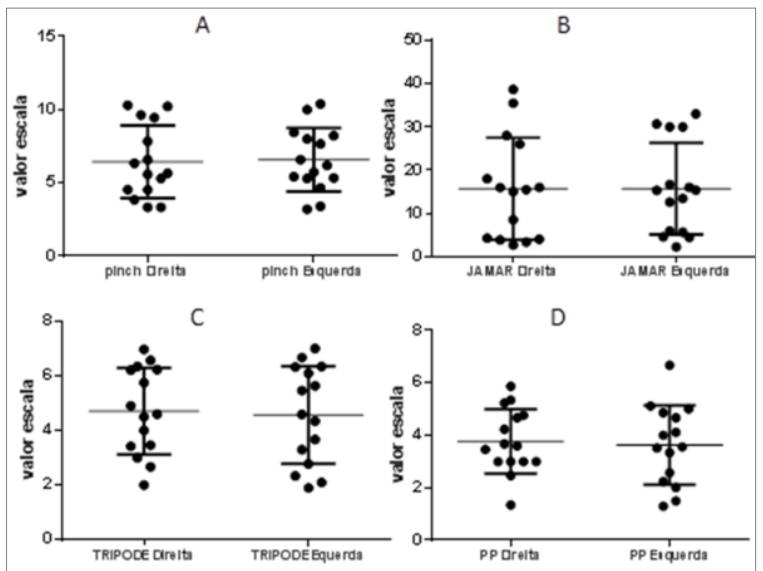

Figura 5: Representação gráfica da avaliação física do lado direito com esquerdo. A: Pinch. B: Jamar. C: Tripode. D: Ponta a Ponta (PP). Fonte: Dados da Pesquisa

Tabela 4. Representação dos resultados encontrados no questionário PRWE

\begin{tabular}{|c|c|c|c|c|c|c|c|c|c|}
\hline \multicolumn{5}{|c|}{ PRÉ-OPERATÓRIO } & \multicolumn{5}{|c|}{ PÓS-OPERATÓRIO } \\
\hline & MX & MN & $\mathbf{M}$ & DP & MX & MN & M & DP & \\
\hline Função & 60 & 5 & 50 & 14,07 & 51 & 4 & 20 & 14,69 & $<0,001$ \\
\hline Dor & 50 & 30 & 46 & 5,78 & 50 & 2 & 25 & 11,38 & $<0,001$ \\
\hline Atividade & 40 & 0 & 40 & 11 & 36 & 12 & 30 & 8,35 & 0,0714 \\
\hline
\end{tabular}

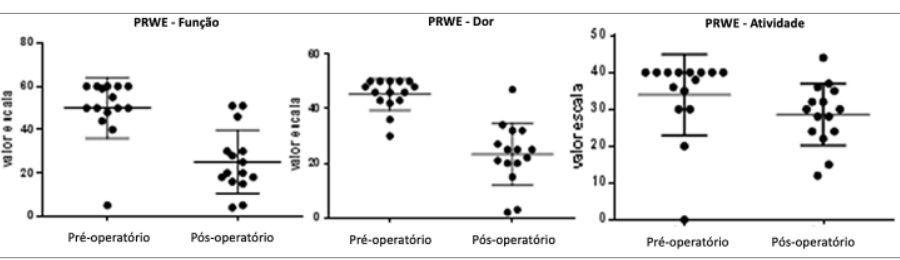

Figura 6: Representação gráfica das variáveis do questionário PRWE, comparando pré com pós-operatório para função, dor e atividade.

DISCUSSÃO

Neste estudo, encontramos resultados satisfatórios $(62,25 \%)$ e consistentes com a literatura para a utilização da técnica cirúrgica Sauvé-Kapandji. Apesar de observar alterações angulares importantes, isso não representou modificação da força entre os membros (Avaliação física) e das atividades realizadas (Questionário PRWE) antes da cirurgia.

Nos últimos anos, vários estudos têm sugerido que o procedimento de SauvéKapandji pode ser um procedimento melhor para o tratamento da osteoartrose póstraumática em pacientes jovens ativos. As indicações para esse procedimento enquadram osteoartrose, impacto ulnocarpal pós-traumático associado a artrose, artrite reumatoide em pacientes jovens com translocação da ulna e alteração articular, artrite reumatoide que necessite de estabilização radioulnar para suportar artroplastia e encurtamento do rádio pós-traumática ou congênita (em casos selecionados) $)^{6,8}$. As principais contraindicações são a artrite séptica ativa, o comprimento insuficiente da ulna, e a ausência da cabeça do radio $^{2,18-20}$.

Os resultados obtidos após tratamento de lesões crônicas da articulação radioulnar distal tem sido inferior a ideal, apesar dos avanços nos estudos da anatomia e biomecânica desta área.

As principais complicações do procedimento Sauvé-Kapandji incluem o retardo de consolidação da artrodese, reossificação do segmento ressecado, instabilidade dolorosa do coto proximal da ulna ou irritação dos implantes. A instabilidade dolorosa do coto proximal da ulna pode causar uma deficiência funcional grave, que pode ser evitada pela estabilização do coto através de uma hemi-fita de tendão, conforme descrito anteriormente. A irritação dos implantes é uma queixa relativamente comum em implantes ulnares ${ }^{18-20}$. Neste estudo, forma observados 6 casos que necessitaram de intervenção cirúrgica por irritação do parafuso. Encontramos.

A técnica de Sauvé-Kapandji para o tratamento dos desarranjos da articulação radioulnar distal não é de aceitação universal. Entretanto, séries maiores têm mostrado que 
este método é eficiente, com uma incidência relativamente baixa de complicações, como observado neste estudo.

A instabilidade estática e dinâmica da ulna no plano radioulnar também foi avaliada pela comparação entre as incidências radiográficas sem carga e com carga, como proposto por Nakamura et al. ${ }^{6}$ Observou-se que alguns casos possuem uma aproximação entre a ulna e o rádio, mesmo sem carga. Todavia, ao fazer o esforço para segurar a carga, não houve redução significativa do espaço, capaz de interferir no resultado.

Apesar desta série ser relativamente pequena e conter casos de desarranjo da articulação radioulnar distal pós-traumáticos, os resultados obtidos, são animadores em relação à aplicabilidade da técnica de Sauvé-Kapandji e da sua eficácia para doenças da articulação radioulnar distal. A literatura acredita que, este procedimento é mais bem indicado para pacientes jovens portadores de sequelas de trauma, evitando procedimentos mais radicais como 0 de Darrach, considerado mais incapacitante. Entretanto, apesar de relativamente simples e de fácil execução, deve ser realizado com precisão para que os bons resultados se repitam ${ }^{17-20}$.

CONCLUSÃO

Concluímos que a técnica de SauvéKapandji produziu resultados satisfatórios e de fácil reprodutibilidade. Porém, séries maiores, contendo grupos controle, ou de comparações com 0 procedimento de Darrach são necessários.

\section{REFERÊNCIAS}

1. Aita MA, Ibanez DS, Saheb GC, Alves RS. Arthroplasty of the distal ulna distal in managing patients with post-traumatic disorders of the distal radioulnar joint: measurement of quality of life. Rev Bras Ortop. 2015;50(6):666-72.

2. Vlček M, Pech J, Musil V, Stingl J. Conservative and Surgical Treatment for Distal Ulna Fractures Associated with Distal Radius Fractures. Acta Chir Orthop Traumatol Cech. 2015;82(6):412-17.

3. Saffar P, Badina A. Treatment of Madelung's deformity. Chir Main. 2015;34(6):279-85.

4. Vedung T, Vinnars B. Resurfacing the distal radioulnar joint with rib perichondrium-a novel method. J Wrist Surg. 2014;3(3):206-10.

5. Lluch A. The sauvé-kapandji procedure. J Wrist Surg. 2013;2(1):33-40.

6. Nakamura T, Ota N, Iwamoto $T$, Sato $K$, Toyama Y. Radiographic parameter analysis on modified sauvé-kapandji procedure. J Wrist Surg. 2013;2(1):19-26.
7. García-López M, Pareja-Esteban JA, Valmañade laSotilla JM, Jiménez-Alcázar LC, MartínezCalvo MA, Plasencia-Arriba MA. [SauvéKapandji procedure in distal radioulnar joint disorders]. Rev Esp Cir OrtopTraumatol. 2013;57(5):340-47.

8. Theodorakis $E$, Fanelli $M$, Ottolenghi $P$, Pappalardo S. An unconventional indication of the Sauve' - Kapandji procedure in a radial shaft pseudoarthrosis and chronic DRUJ dislocation: a case report. Clin Ter. 2013;164(2):e133-36.

9. Jacoby SM, Bachoura A, Diprinzio EV, Culp $\mathrm{RW}$, Osterman AL. Complications following one-bone forearm surgery for posttraumatic forearm and distal radioulnar joint instability. $J$ Hand Surg Am. 2013;38(5):976-82.

10. Condamine JL, Lebreton L, Aubriot JH. [The Sauvé-Kapandji operation. Analysis and results of 69 cases]. Ann Chir Main Memb Super. 1992;11(1):27-39.

11. Kapandji Al. [Distal radio-ulnar prosthesis]. Ann Chir Main Memb Super. 1992;11(4): 320-32.

12. Kapandji IA. The Kapandji-Sauvé operation. Its techniques and indications in non rheumatoid diseases. Ann Chir Main. 1986;5(3):181-93.

13. Sebastin SJ, Larson BP, Chung KC. History and evolution of the Sauvé-Kapandji procedure. J Hand Surg Am. 2012;37(9):1895-902.

14. Lluch A. The Sauvé-Kapandji procedure: indications and tips for surgical success. Hand Clin. 2010;26(4):559-72.

15. Haferkamp H. [Kapandji-Sauvé procedure with distal radioulnar fusion and segmental resection of the ulna]. Oper Orthop Traumatol. 2012;24(1):13-22.

16. Luchetti $R$, Khanchandani $P$, Da Rin F, Borelli PP, Mathoulin C, Atzei A. Arthroscopically assisted Sauvé-Kapandji procedure: an advanced technique for distal radioulnar joint arthritis. Tech Hand UpExtrem Surg. 2008;12(4):216-20.

17. Ross M, Thomas J, Couzens G, Coleman S. Salvage of the unstable Sauvé-Kapandji procedure: a new technique. Tech Hand UpExtrem Surg. 2007;11(1):87-92.

18. Fujita S, Masada K, Takeuchi E, Yasuda M, Komatsubara Y, Hashimoto H. Modified SauveKapandji procedure for disorders of the distal radioulnar joint in patients with rheumatoid arthritis. Surgical technique. J Bone Joint Surg Am. 2006;88(1):24-8.

19. Kapandji Al. [Technical improvement of the Kapandji-Sauvé operation, called "Technique III"]. Ann Chir Main Memb Super. 1998;17(1):78-86.

20.Gaulke R. [Sauvé-Kapandji procedure in destructive monarthritis of the distal radioulnar joint]. Z Rheumatol. 2003; 62(5):468-73. 


\section{CONFLITO DE INTERESSES}

Os autores declaram não haver conflitos de interesse

\section{AUTOR PARA CORRESPONDENCIA}

\section{Caroline Brum Sena}

Av. Bandeirantes, 3900 - Vila Monte Alegre,

14049-900 Ribeirão Preto - SP, Brasil

E-mail: dra.caroline.sena@gmail.com

Submetido em 31/03/2020

Aceito em 19/05/2020 